\title{
Utilization of the sol-gel technique for the development of novel stationary phases for capillary electrochromatography on a chip
}

\author{
S. Constantin ${ }^{\mathrm{a}}$, R. Freitag ${ }^{\mathrm{a},}$, D. Solignac ${ }^{\mathrm{b}}$, A. Sayah ${ }^{\mathrm{b}}$, M.A.M. Gijs ${ }^{\mathrm{b}}$ \\ ${ }^{a}$ Department of Chemistry, Center of Biotechnology, Swiss Federal Institute of Technology, \\ Lausanne 1015, Ecublens, Switzerland \\ ${ }^{\mathrm{b}}$ Institute of Microsystems, Swiss Federal Institute of Technology, \\ Lausanne 1015, Ecublens, Switzerland
}

\begin{abstract}
Capillary electrochromatography (CEC) appears ideally suited for high performance separations at small scale, i.e. on a chip. Problems with the reproducible production of the required $\mu$-HPLC column, but also the lack of commercially available $\mu$-CEC instruments have prevented many putative applicants of this promising technique from entering the field. In this paper, a fast and easy way to produce selfcontaining open-tubular $\mu$-CEC columns $\left(\mathrm{C}_{8}\right.$-moieties for reversed phase applications) by the sol-gel technique is described. The corresponding chips were designed to be compatible with a commercial system for capillary electrophoresis (namely a Beckman P/ACE 5500 system with diode array detection). Method development and application hence benefited from the injection and the detection options of this setup. The separation of a mixture of three uncharged analytes (polycyclic aromatic hydrocarbons) by the chip is given as example. Under optimized conditions, the performance of the chip appeared to be comparable or better than that of capillary-based CEC columns of the same kind. (C) 2001 Elsevier Science B.V. All rights reserved.
\end{abstract}

Keywords: Sol-gel technique; Capillary electrochromatography (CEC); Stationary phase; Microchip

\section{Introduction}

In recent years, microfabricated devices ('microchips') for bio-chemical and bio-analytical applications have been shown to be more than just an alternative to conventional (macroscopic) techniques. In the field of analytical separations, microchips have been successfully employed for free zone electrophoresis [1-3] and gel electrophoresis [4]. Capillary electrochromatography (CEC) is an emerging, very promising new option for microseparation and analysis of biologicals, which conceivably also would profit tremendously from being realized in a microfluidic system ( $\mu$-CEC), since in this case mobile phase gradients, flow control units and multidimensional separations could be realized much more easily than in the capillary electrophoresis (CE)-systems used at present to perform CEC. It must be acknowledged, however, that $\mu$-CEC will have to overcome some important technological hurdles, including the need for dedicated instrumentation and suitable columns/stationary phases before electrochromatography and

\footnotetext{
${ }^{*}$ Corresponding author. Tel.: +41-2169-36108; fax: +41-2169-36030. E-mail address: ruth.freitag@epfl.ch (R. Freitag).
}

multidimensional electrochromatography $\left(\mu-\mathrm{CEC}^{\mathrm{n}}\right)$ on a chip becomes a viable option in analytical chemistry.

In CEC in general, the separation of charged and noncharged molecules is based on different affinities to a stationary phase or different distributions between a stationary and a mobile phase. The mobile phase is pumped by electroosmosis. Putatively very high column efficiencies and hence superior separations could be obtained, while very small sample volumes should be sufficient for a reliable analysis. The column/stationary phase is rather important in CEC, since it is responsible not only for the separation, but also for the electroosmotic flow (EOF), i.e. the pumping of the mobile phase. The question of how to realize a chromatographic column inside a capillary (or on a chip) is by no means answered. Most authors seem to prefer an HPLC-type particle bed, even though the corresponding packing procedure is known to be difficult [5-7]. Tapered capillaries have been suggested to avoid the use of frits in such a setup [8].

Our research groups has in the past four years designed and investigated several novel types of self-containing (open-tubular and macroporous monolithic) stationary phases for CEC $[9,10]$. In this paper the transfer of the open-tubular approach to the chip-format is described. 


\section{Experimental}

\subsection{Materials and chemicals}

Tetraethoxysilane (TEOS), $n$-octyltriethoxysilane $\left(\mathrm{C}_{8^{-}}\right.$ TEOS), polycyclic aromatic hydrocarbons (PAH) (naphthalene, phenanthrene, pyrene), hydrochloric acid, sodium hydroxide were from Fluka (Buchs, Switzerland). Acetonitrile (ACN, HPLC grade) was purchased from Biosolve Ltd. (Valkenswaard, The Netherlands). Water was purified using an Elix-3 system (Millipore, Bedford, MA, USA). Fused silica capillaries were from Polymicro Technologies (Phoenix, AR, USA).

\subsection{Microchips}

Microchips were microfabricated from pyrex-wafers (pyrex borosilicate, glass code 7740). A Photolithographic process followed by a HF etching step was used to create a channel on the chip, as described in detail elsewhere [11]. Briefly, the pyrex wafer was coated with a chromium layer $(50 \mathrm{~nm})$, a gold layer $(200 \mathrm{~nm})$ and the photoresist. After the exposition and the development of the photoresist, the metal layers were structured. The microchannels were etched using a $49 \%$ HF solution. Finally, all the layers were removed. The patterned pyrex wafer was sawed into the individual chips. After etching, the channels were sealed by thermal fusion bonding $\left(650^{\circ} \mathrm{C}\right)$ with a Pyrex cover plate, Fig. 1. The separation channels dimensions were as follows: width $90 \mu \mathrm{m}$, depth $23 \mu \mathrm{m}$, length $60 \mathrm{~cm}$. The inlet and the outlet holes of the chips were subsequently enlarged by powder blasting [12]. Silica capillaries were inserted into these cavities using an in-house technique designed to minimize any dead volume, as suggested by Bings et al. [13]. Epoxy glue (EPOTEK 353 NDT) was used to fix the capillaries in their position.

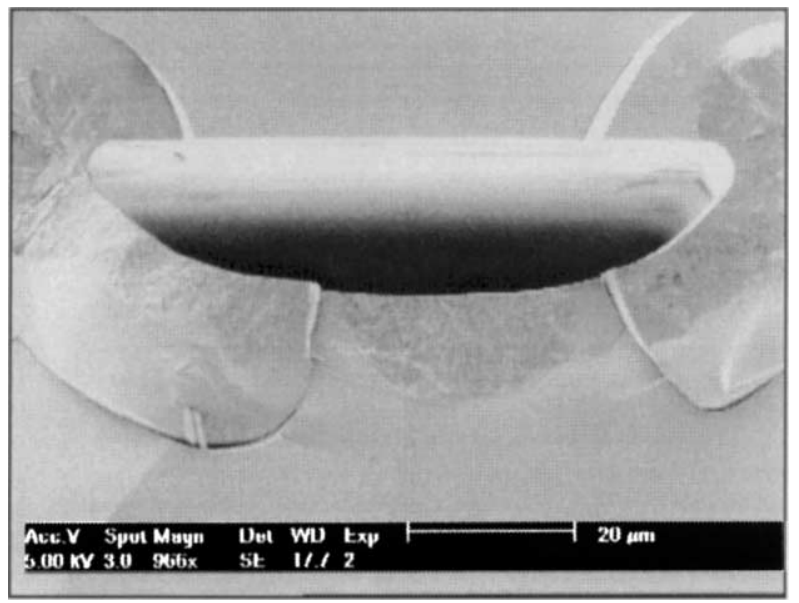

Fig. 1. SEM microphotograph of a chip channel $(\times 966)$, applied voltage $5 \mathrm{kV}$, working distance 17.7, sample sputtered with a gold layer.

\subsection{Stationary phases}

For the preparation of the stationary phase $282 \mu \mathrm{l}$ of $\mathrm{C}_{8^{-}}$ TEOS, $500 \mu \mathrm{l}$ of TEOS, corresponding to a 1.0:2.5 molar ration, and $177 \mu \mathrm{l}$ of ethanol were placed in an Eppendorf vial. The solution was briefly vortexed, then $52 \mu \mathrm{l}$ of water and $53 \mu \mathrm{l}$ of $\mathrm{HCl}(1 \mathrm{M}$, catalyst) were added. The mixture was vortexed at $2500 \mathrm{rpm}$ for $1 \mathrm{~min}$ and allowed to react for an additional $10 \mathrm{~min}$. Although the sol-gel process had started, the solution was still liquid at this point and could be injected via the inlet capillary into the chip-channel using a syringe with a plastic fitting. Injection was stopped when the liquid reached the microchip outlet. After $10 \mathrm{~min}$ of reaction within the chip's channel, the excess solution was withdrawn by syringe via the inlet capillary. The vacuum was left in place for at least two hours. The microchip was subsequently dried in a vacuum oven $(20 \mathrm{mbar})$ at $30^{\circ} \mathrm{C}$, for $2 \mathrm{~h}$, then flushed with ACN ( $5 \mathrm{~min})$, followed by a $10^{-2} \mathrm{M}$ aqueous sodium hydroxide solution (alkaline wash, $10 \mathrm{~min}$ ). The basic solution was then removed by syringe. Prior to use, the chips were mounted into the standard capillary cartridge of the Beckman P/ACE 5500 system, Fig. 2. The detection slit $(800 \mu \mathrm{m} \times 100 \mu \mathrm{m})$ of the cartridge was aligned with the outlet of the chip's separation channel to allow for detection. The cooling system of the cartridge adapted to house a chip was short-circuited to avoid leakage of the cooling liquid. CEC-columns in fused silica capillaries were prepared as described elsewhere [9] and used for comparison.

\subsection{CEC-measurements}

The Beckman P/ACE system 5500 with diode array detection was used throughout the CEC experiments both with the chip-based and with the fused silica capillary based

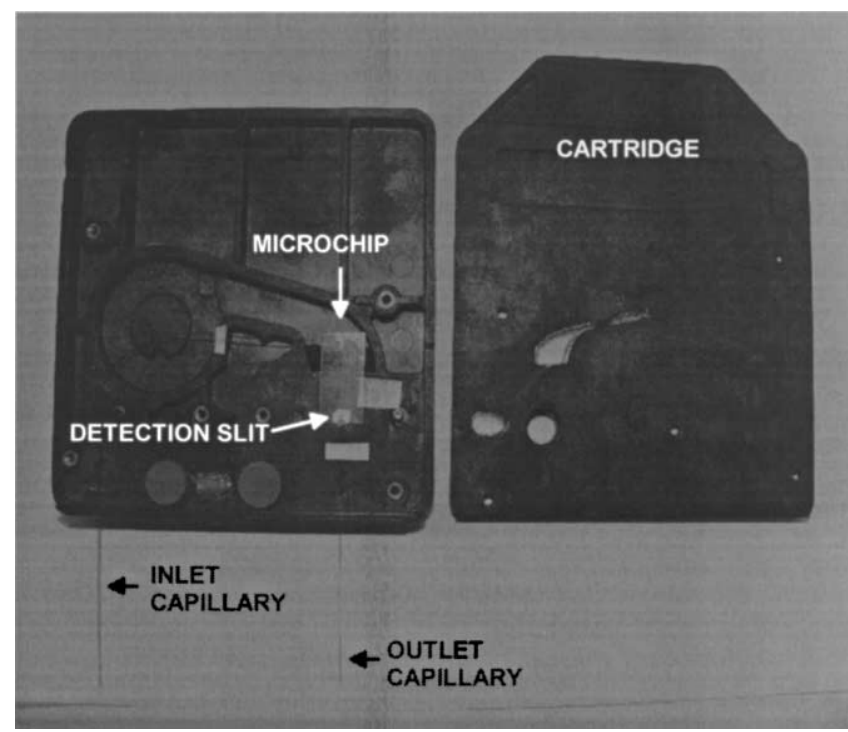

Fig. 2. Photograph of a chip mounted into the standard capillary cartridge. 
columns. The injection, voltage supply, and detection facilities of this system were used in the conventional manner to perform CEC on the chips.

Before the first run, chips were flushed for 5 min with $\mathrm{ACN}$, followed by $15 \mathrm{~min}$ with the mobile phase (unless mentioned otherwise $\mathrm{ACN} / \mathrm{H}_{2} \mathrm{O} 1: 1 \mathrm{v} / \mathrm{v}$, containing $5 \mathrm{mM}$ of $\mathrm{NaCl}$ ). The chips were equilibrated for $1 \mathrm{~h}$ with mobile phase, then a voltage of $15 \mathrm{kV}$ was applied for $30 \mathrm{~min}$. Blank runs were carried out until the baseline became stable. Samples were injected hydrodynamically into the inlet capillary (unless mentioned otherwise $3 \mathrm{~s}, 1.36 \mathrm{bar}$ ). A detection wavelength of $280 \mathrm{~nm}$, a temperature of $25^{\circ} \mathrm{C}$ and a voltage of $15 \mathrm{kV}$ (unless mentioned otherwise) were adjusted. A mixture containing three uncharged polycyclic aromatic hydrocarbons, namely naphthalene, phenanthrene, and pyrene, $10 \mathrm{mM}$ each, was used as sample.

\section{Results and discussion}

\subsection{Preparation of the chip}

Previous investigations with capillaries of different diameter had shown that the synthesis protocol has to be adapted in terms of monomer composition and concentration, as well as in terms of the water content and the temperature and the $\mathrm{pH}$ of the reaction mixture to the geometry, mainly the diameter, of the capillary in order to reproducibly obtain the desired mechanically and chemically stable stationary phases [9]. These factors together with the duration allowed for the reaction prior to injection into the capillary, affect the structure and the viscosity of the gel and hence the outcome of the stationary phases installation inside the capillary. The protocol used here for stationary phase synthesis, see below, was the result of a similar optimization procedure for the chip channel dimensions. A major advantage of using a home-made chip rather than a commercially available fused silica capillary, was the fact that the channel geometry could to some extent be adapted to the stationary phase synthesis and not vice versa. Thus synthesis conditions, e.g. the temperature, could be adjusted to the limitations posed by the various system components. One major limitation did arise from the necessity to connect inlet and outlet capillaries to the chip in order to make it compatible to the conventional CE-system, Fig. 3. Special attention has to be paid to the glue chosen to connect the chips and the capillaries, since this glue had to remain chemically inert during stationary phase synthesis and subsequent applications of the CEC-chip. The epoxy-glue technique described in the experimental section was found to fulfill these requirements.

\subsection{Preparation of the stationary phase}

The aim of the stationary phase (column) synthesis for CEC is to produce a material, which exhibits differential

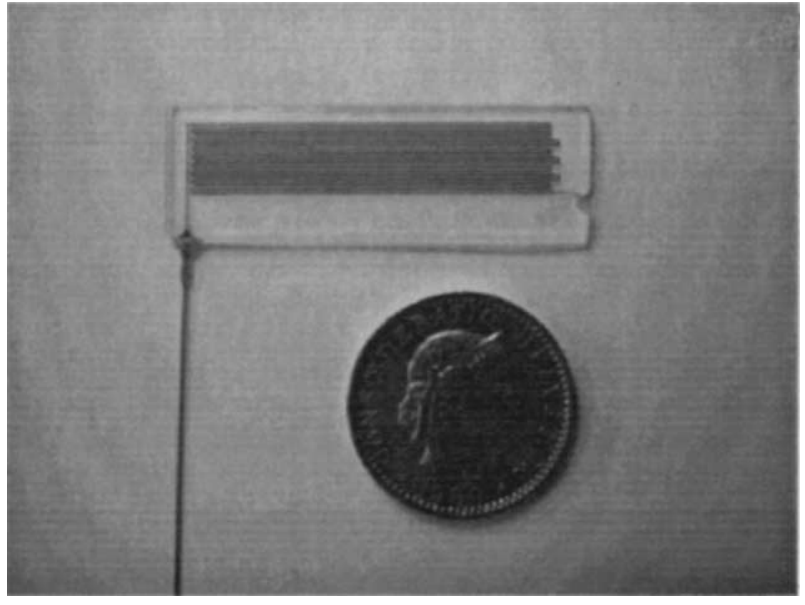

Fig. 3. Photograph of a microchip with an inlet connection to a silica capillary. The channel is made visible by injection of blue ink.

interactions with the constituents of the sample to be analyzed. In our case, a reversed phase-type of interaction was desired, since the goal was the separation of a mixture of uncharged, nonpolar substances. In fused silica capillaries, such stationary phases have been created in the form of organically modified silicate (ormosil) coatings [9]. Ormosils are obtained via the sol-gel technique [14]. This technique calls for a complex hydrolysis/condensation process of suitable metalorganic precursors, Fig. 4. Using TEOS and alkylethoxysilane (alkyl-TEOS) as co-monomers, stationary phases containing silanol groups as well as organic moieties were produced. The silanol groups will later generate the EOF (i.e. the "pump"), whereas the organic moieties mediate the chromatographic separation.

Stationary phases containing $\mathrm{C}_{8}$-TEOS were prepared in the channel of the microchip as described in the experimental section. Initial conditions in the sol-gel process were chosen in such a way that a liquid was obtained, containing a high amount of unreacted silanol groups ( $\mathrm{Si}-\mathrm{OH})$. This liquid could still be injected hydrodynamically into the future separation channel. Inside the hydrolysis/condensation process was allowed to proceed further, involving this time also the silanol groups typically found on the surface of the channel and the remaining silanol groups of the organic/ inorganic ormosil cluster. The covalent links thereby formed between the gel structure and these surface groups are responsible for anchoring the ormosil-based stationary phase securely to the channel surface.

The silanol group density of the channel/capillary surface is hence important for the stability of the future column and in the case of conventional fused silica capillaries sometimes has to be increased by treatment with an alkaline solution [15]. Since Pyrex rather than fused silica was used as support in case of the CEC-chips, the silanol group density was expected to be much higher and already sufficient for stable stationary phase bonding. The lengthy alkaline treatment was thus omitted without any noticeable disadvantageous 


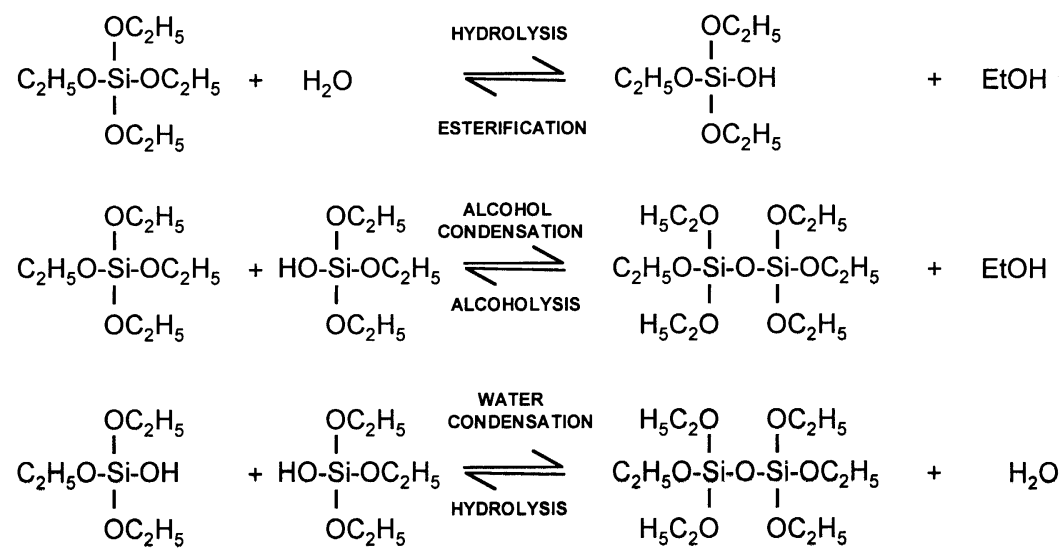

Fig. 4. Chemical reactions involved in the sol-gel process. When one part of TEOS is replaced by alkylalkoxysilane $\left[\mathrm{R}_{a}-\operatorname{Si}-\left(\mathrm{OR}^{\prime}\right)_{b}(a=1-3, b=4-a)\right]$, organically modified silicates (ormosils) are obtained.

effects for the stationary phase stability. Some minutes after the liquid had been injected, the channel was flushed with a gas stream. As a result, most of the ormosil-gel was removed, while a thin coating of stationary phase remained on the inner wall of the separation channel as seen in Fig. 5. To obtain this picture, a fluorescent dye (FITC) was incorporated into the ormosil, hence the intensity of the fluorescent light at the sides is much higher (channel depth $23 \mu \mathrm{m})$ than that of either the top or the bottom of the channel, where only the thin ormosil layer is found.

One of the main problems in $\mu$-CEC is the low mass loadability of the stationary phase, which directly results from the miniaturized format of the separation channel [16]. However, as previously shown [9], the inclusion of an alkaline wash into the sol-gel procedure results in a pronounced wrinkling of the stationary phase surface and hence an increased capacity. Presumably, a similar improvement was also possible in case of the CEC-chip.

\subsection{Electrochromatographic separations}

The mobile phase is of obvious importance in CEC. It must dissolve the sample components, but also has to allow for the generation of a sufficient electroosmotic flow. In our

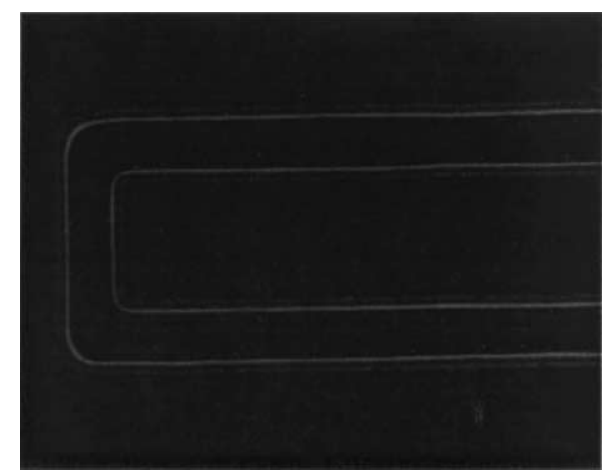

Fig. 5. Optical microphotograph of a microchip containing a fluorescent dye $(\times 50)$, UV light. case, special attention during mobile phase selection had in addition to be paid to the microchip's connections to the inlet and outlet capillaries, since the glue had to be chemically inert towards the chosen mobile phase. In the end, a classical mobile phase for reversed phase chromatography was chosen, which consisted of a 1:1 (v/v) mixture of acetonitrile and water, containing in addition $5 \mathrm{mM} \mathrm{NaCl}$ to avoid breakdown of the electrical current during the run [17].

Pyrex tends to show a noisy baseline after being irradiated with UV light for some time. This limited the detection wavelengths suitable for the CEC-chip experiments to values $>270 \mathrm{~nm}$ (in our case: $280 \mathrm{~nm}$ ). This may in the future pose a problem for some analytes. However, for the prove-of-feasibility intended here, a mixture of uncharged, hydrophobic polycyclic aromatic hydrocarbons (PAH) was chosen as standard sample for the CEC-experiments. These compounds, namely naphthalene, phenanthrene and pyrene, all absorb above $270 \mathrm{~nm}$ and hence were easily detected in our setup. Moreover, since the chosen analytes were not electrically charged, any separation observed could not by due to electrophoresis and hence proved that a chromatographic separation mechanism was responsible.

In order to allow a comparison of the performance, the ormosil stationary phase was prepared in both a standard fused silica CE-capillary and a Pyrex microchip. The results obtained with both column-types for the separation of the standard mixture were subsequently compared. Figs. 6 and 7 show the results obtained in a capillary and in a chip, respectively.

Naphthalene, phenanthrene and pyrene separated in the fused silica capillary all gave comparatively broad peaks with elution times ranging from 9.5 to $13 \mathrm{~min}$. The same compounds analyzed in the chips give somewhat sharper peaks with shorter elution times between 6.0-6.5 min. The resolution is better in case of the capillary. However, since base-line separation is achieved in both cases, this is of no analytical advantage. Plate numbers (chromatographic efficiency) are superior in case of the chip. However, since the mobile phase composition, the applied electric field and the 


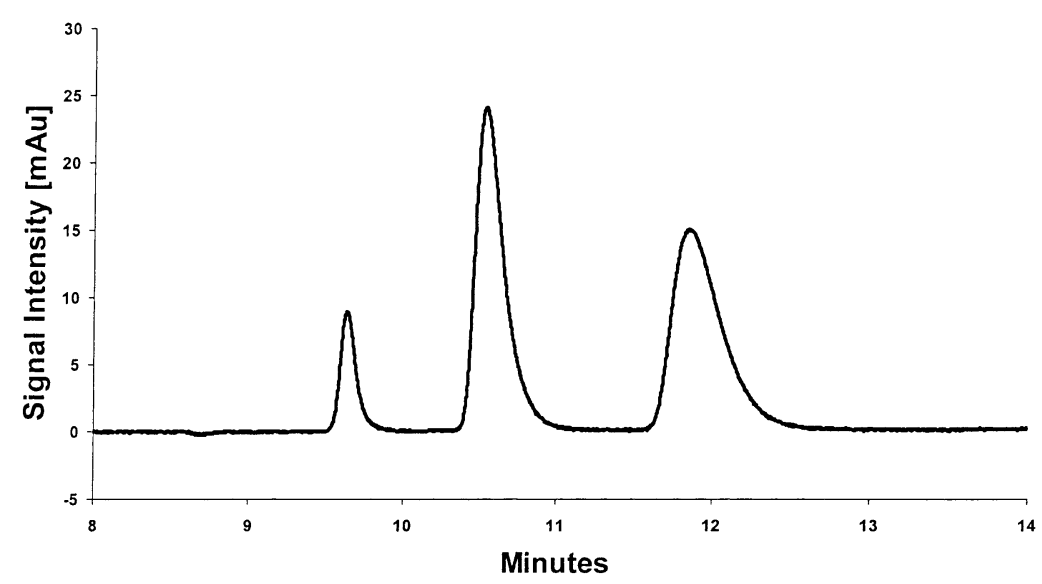

Fig. 6. Electrochromatogram of a mixture of PAHs in a coated fused silica capillary. Elution order: naphthalene, phenanthrene, pyrene (sample concentration, each $10 \mathrm{mM}$ ). The separation is performed in a fused silica capillary coated with a reversed phase made through the described sol-gel process (molar ratio $\mathrm{C}_{8}$-TEOS/TEOS 1.0:2.5). Mobile phase: acetonitrile/water $(1: 1 \mathrm{v} / \mathrm{v})$ containing $5 \mathrm{mM} \mathrm{NaCl}$, voltage $12.5 \mathrm{kV}$; hydrodynamic injection: $1 \mathrm{~s}$ at 1.36 bar; detection wavelength $280 \mathrm{~nm}$; temperature $25^{\circ} \mathrm{C}$; capillary dimensions: length $40 \mathrm{~cm}$, inner diameter $50 \mu \mathrm{m}$.

injection time are slightly different, a direct comparison of these values is not admissible. If anything, the separation achieved with the CEC-chip appears to be of equal quality of even slightly superior to the one obtained with the conventional capillary under optimized conditions. This could be due to the diffusion path length confronting the analytes. The fused silica capillary had a constant diameter of $50 \mu \mathrm{m}$, which is about twice as much as the depth of

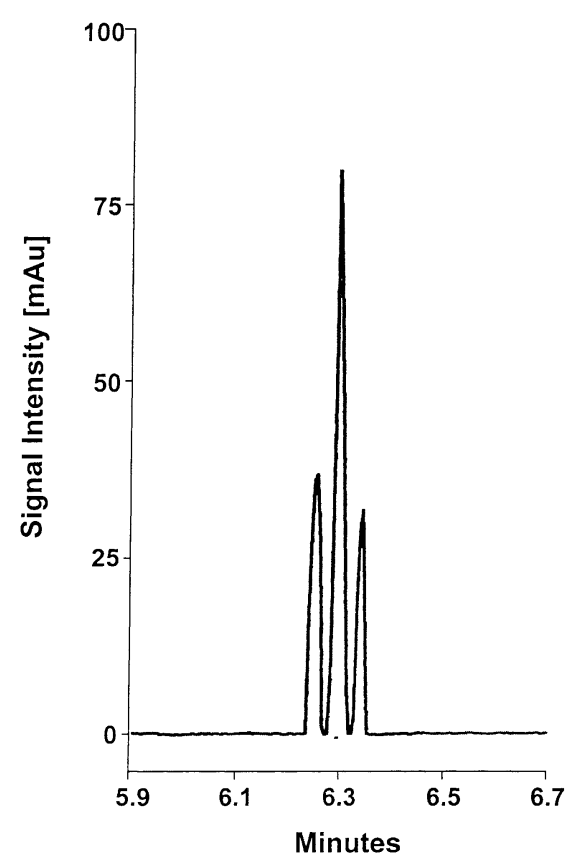

Fig. 7. Electrochromatogram of a mixture of PAHs in a coated pyrex chip. Elution order: naphthalene, phenanthrene, pyrene. Sample and stationary phase as in Fig. 6. Mobile phase: acetonitrile/water (1:1 v/v) containing $5 \mathrm{mM} \mathrm{NaCl}$, voltage $15 \mathrm{kV}$; hydrodynamic injection: $3 \mathrm{~s}$ at 1.36 bar; detection wavelength $280 \mathrm{~nm}$; temperature $25^{\circ} \mathrm{C}$; dimensions of the chip channel: width $90 \mu \mathrm{m}$, depth $23 \mu \mathrm{m}$, length $60 \mathrm{~cm}$. Length of the connected capillaries: inlet $15 \mathrm{~cm}$, outlet $7 \mathrm{~cm}$. the chip channel $(23 \mu \mathrm{m})$. An analyte in the middle of a given separation channel will be in closer proximity of a wall in the case of the CEC-chip than in the case of the CEC-capillary.

A major worry prior to the application of the CEC-chip was the possibility of peak disturbance due to pronounced Joule heating. This could have been a problem with the chip rather than with the conventional capillary, since in this case, the cooling liquid flow of the cartridge had to be shortcircuited. However, from the results shown in Fig. 7, this seems not to be the case. The use of a hydro-organic mobile phase can be assumed to be largely responsible for this fact, since the Joule heating effect is greatly reduced under these circumstances [18].

\section{Conclusions}

The sol-gel technique allows the reproducible preparation of CEC-microcolumns. The columns are mechanically stable and no frits or special structures are needed to retain them in place. The resolution obtained for a typical standard mixture of uncharged substances is of similar quality as that obtained in the case of conventional capillary columns of the same type.

\section{References}

[1] A. Manz, D.J. Harrison, E. Verpoorte, J.C. Fettinger, A. Paulus, H. Lüdi, H.M. Widmer, Planar chips technology for miniaturization and integration of separation techniques into monitoring systems, J. Chromatogr. 593 (1992) 253.

[2] K. Seiler, D.J. Harrison, A. Manz, Planar glass chips for capillary electrophoresis - repetitive sample injection, quantitation, and separation efficiency, Anal. Chem. 65 (1993) 1481.

[3] A.T. Woolley, R.A. Mathies, Ultra-high-speed DNA-sequencing using capillary electrophoresis chips, Anal. Chem. 67 (1995) 3676. 
[4] S.C. Jacobson, R. Hergenröder, L.B. Koutny, J.M. Ramsey, Effects of injection schemes and column geometry on the performance of microchip electrophoresis devices, Anal. Chem. 66 (1994) 2369.

[5] G. Ocvirk, Verpoorte, E. et al. Anal. Methods Instrum. 2 (1995) 74.

[6] M.T. Dulay, R.P. Kulkarni, R.N. Zare, Preparation and characterization of monolithic porous capillary columns loaded with chromatographic particles, Anal. Chem. 70 (1994) 5103-5107.

[7] Q. Tang, B. Xin, M.L. Lee, Monolithic columns containing sol-gel bonded octadecylsilica for capillary electrochromatography, J. Chromatogr. A 837 (1999) 35-50.

[8] M. Mayer, E. Rapp, C. Mark, G.J.M. Bruin, Fritless capillary electrochromatography, Electrophoresis 20 (1999) 43-49.

[9] S. Constantin, R. Freitag, Preparation of stationary phases for opentubular capillary electrochromatography using the sol-gel method, J. Chromatogr. A 887 (1/2) (2000) 253-263.

[10] D. Hoegger, R. Freitag, Acrylamide-based monoliths as robust stationary phases for capillary electrochromatography, J. Chromatogr. A 914 (2001) 211-222.

[11] S. Sayah, D. Solignac, T. Cueni, M.A.M. Gijs, Development of novel low-temperature bonding technologies for microchip chemical analysis applications, Sens. and Actuators, in press.
[12] E. Belloy, S. Thurre, E. Walckiers, A. Sayah, M.A.M. Gijs, The introduction of powder blasting for sensor and microsystem applications, Sen. and Actuators A 84 (2000) 330-337.

[13] N.H. Bings, C. Wang, C.D. Skinner, C.L. Colyer, P. Thibault, D.J. Harrison, Microfluidic devices connected to fused-silica capillaries with minimal dead volume, Anal. Chem. 71 (15) (1999) 3292-3296.

[14] C.J. Brinker, G.W. Scherer, Sol-Gel Science, The Physics and Chemistry of Sol-Gel Processing, Academic Press Limited, New York, 1990, ISBN 0-12-134970-5.

[15] J.H. Knox, M.T. Gilbert, J. Chromatogr. 186 (1979) 405.

[16] J.J. Pesek, M.T. Matyska, Electrochromatography in chemically modified etched fused-silica capillaries, J. Chromatogr. A $736(1 / 2)$ (1996) 255-264.

[17] S. Cherkaoui, J.L. Veuthey, Development and robustness testing of a nonaqueous capillary electrophoresis method for the analysis of nonsteroidal anti-inflammatory drugs, J. Chromatogr. A 874 (2000) $121-129$

[18] P.B. Wright, A.S. Lister, J.G. Dorsey, Behavior and use of monaqueous media without supporting electrolyte in capillary electrophoresis and capillary electrochromatography, Anal. Chem. 69 (1997) 3251-3259. 